\title{
Examining the impact of scale variations on soil moisture downscaling using temporal persistence
}

\author{
$\underline{\text { Gift Dumedah }}^{\mathrm{a}}$, Jeffrey P. Walker ${ }^{\mathrm{a}}$, and Benjamin Mililli ${ }^{\mathrm{a}}$ \\ ${ }^{a}$ Department of Civil Engineering, Monash University, Melbourne, VIC, Australia, 6845 \\ Email: gift.dumedah@monash.edu.au
}

\begin{abstract}
Soil moisture estimation is an integral component of operational hydrology including flood forecasting, drought monitoring, catchment management, and groundwater recharge estimation. Typically, soil moisture information is required at variable spatial scales for the above applications. As a result, soil moisture data at one spatial scale are often aggregated to larger areas or disaggregated to smaller areas for a particular application. There is also increasing demand for accurate downscaling of satellite derived data to smaller spatial resolutions. This transition in spatial scale is often fraught with uncertainties because of the high spatiotemporal variability of soil moisture. However, the rank of soil moisture at a particular location in relation to other spatial locations is widely known to be temporally stable at a certain probability. The concept of temporal persistence of soil moisture offers the potential to better estimate soil moisture across spatial scales. The application of temporal persistence for estimation of soil moisture across spatial scales has not been thoroughly investigated in the literature. As a result, this study examines the relationship between the rank stability of soil moisture and physiographic features including vegetation types and soil texture groups. This investigation provides physiographic explanations for temporal persistence of soil moisture, and therefore facilitate soil moisture estimation across scales.
\end{abstract}

To assess the estimation across scales, soil moisture data for three spatial resolutions of $12-k m, 5-k m$, and 1$\mathrm{km}$ data for the Yanco region in New South Wales, Australia were used. The soil moisture data for the 12- $\mathrm{km}$, 5- $\mathrm{km}$ and 1- $\mathrm{km}$ resolutions are land surface model estimates obtained from the Joint UK Land Environment Simulator (JULES). The vegetation data set was obtained from the Australian National Dynamic Land Cover Data set (DLCD). The DLCD was generated from a 16-day Enhanced Vegetation Index composite collected at 250- $m$ resolution from Moderate Resolution Imaging Spectro-radiometer (MODIS) for the period from 2000 to 2008. The DLCD has land cover features clustered into 34 ISO classes with descriptions for the structural character of vegetation, ranging from cultivated and managed land covers (crops and pastures) to natural land covers such as closed forest and sparse, open grasslands.

The soil texture data set was derived from the Digital Atlas of Australian Soils which was obtained from the Australian Soil Resource Information System (ASRIS). ASRIS provides a digital map of soil types and their descriptions, typical ranges for soil properties for each soil type, morphology, and physical properties of soil profiles. The soil classification in ASRIS has six main textural groups including sands, sandy loams, loams, clay loams, clay, and light clays. Moreover, the meteorological forcing data including short and long wave incoming radiation, air temperature, precipitation, wind speed, pressure, and specific humidity were obtained from the Australian Community Climate Earth-System Simulator - Australian (ACCESS-A) at hourly time step with approximately $12-\mathrm{km}$ spatial resolution. The ACCESS-A precipitation data set was bias corrected using a 5- $\mathrm{km}$ gridded precipitation data from the Australian Water Availability Project (AWAP) data obtained through the Australian Bureau of Meteorology.

The results identified temporally stable locations between the three resolution pairs with a percent relative difference of about $6 \%$ maximum and $0.1 \%$ minimum; indicating that the overall difference for downscaling soil moisture across the three resolution pairs is about $3 \%$ of the original value. It was found that temporal stability of soil moisture can facilitate downscaling of soil moisture across different spatial scales, with improved accuracy for decreasing size of spatial resolution. Soil texture and vegetation land cover do not provide strong indicators to identify temporally stable locations, with vegetation cover having a higher correlation than soil texture because of its high homogeneity in the Yanco area.

Keywords: Soil moisture, Temporal persistence, Downscaling. 


\section{INTRODUCTION}

Satellite soil moisture data sets are increasingly been made available at varied spatial resolutions for use in several applications. For practical hydrologic use, an understanding of soil moisture scaling across multiple spatial scales is needed to effectively use the data at a specific spatial resolution. The scaling of soil moisture across spatial scales is plagued with challenges, mainly due to the high space-time variability of soil moisture [Cosh et al., 2008; Martinez et al., 2008; Western et al., 2004, 2002]. An established property of soil moisture is that soil moisture at a specific spatial location will usually remain persistent in relation to soil moisture at other locations through time [Vachaud et al., 1985]. This unique property is referred to as temporal persistence or rank stability of soil moisture [Chen, 2006; Vachaud et al., 1985]. This property has been widely recognised by Martinez et al. [2008]; Cosh et al. [2008]; and Dumedah and Coulibaly [2011], showing that the rank of soil moisture at a particular location in relation to other spatial locations is temporally stable at a certain probability.

While numerous studies have examined scaling of soil moisture [Martinez et al., 2008; Cosh et al., 2008; Martinez Fernandez and Ceballos, 2005; Western et al., 2004], limited studies have actually employed temporal persistence to downscale soil moisture across spatial scales. As a result, this study uses the temporal stability of soil moisture in a trading-space-for-time analysis [Singh et al., 2011] by using the rank persistence of soil moisture in space for extrapolation in time. This study investigates whether rank stability of soil moisture can be applied as a basis for downscaling, by using three overlapping soil moisture data at three different spatial resolutions: $12-\mathrm{km}, 5-\mathrm{km}$, and 1- $\mathrm{km}$. The rank stability facilitates the determination of temporally stable locations across the three spatial resolutions, which in turn are examined in relation to physiographic variations based on soil texture and land cover data. Evaluation of these relationships is important to improve the development of soil moisture downscaling procedures across spatial scales.

\section{MATERials AND METHODS}

\subsection{Experimental site, and data sets}

The experimental site selected for the study is the Yanco area, located within the Murrumbidgee Catchment in New South Wales, Australia (see Figure 1). The Yanco area was chosen because of its long record of in-situ soil moisture data available through the OzNet program (www.oznet.org.au), with the potential for future evaluation of the downscaled data against the in-situ data. The area is relatively flat and characterized with pasture, minimal wooded vegetation and agricultural crops with rice and barley in main rotation [Smith et al., 2012]. The semi-arid conditions of the area are accompanied by finer textured soils characteristic of the western plains of the catchment [Peischl et al., 2012; Smith et al., 2012].

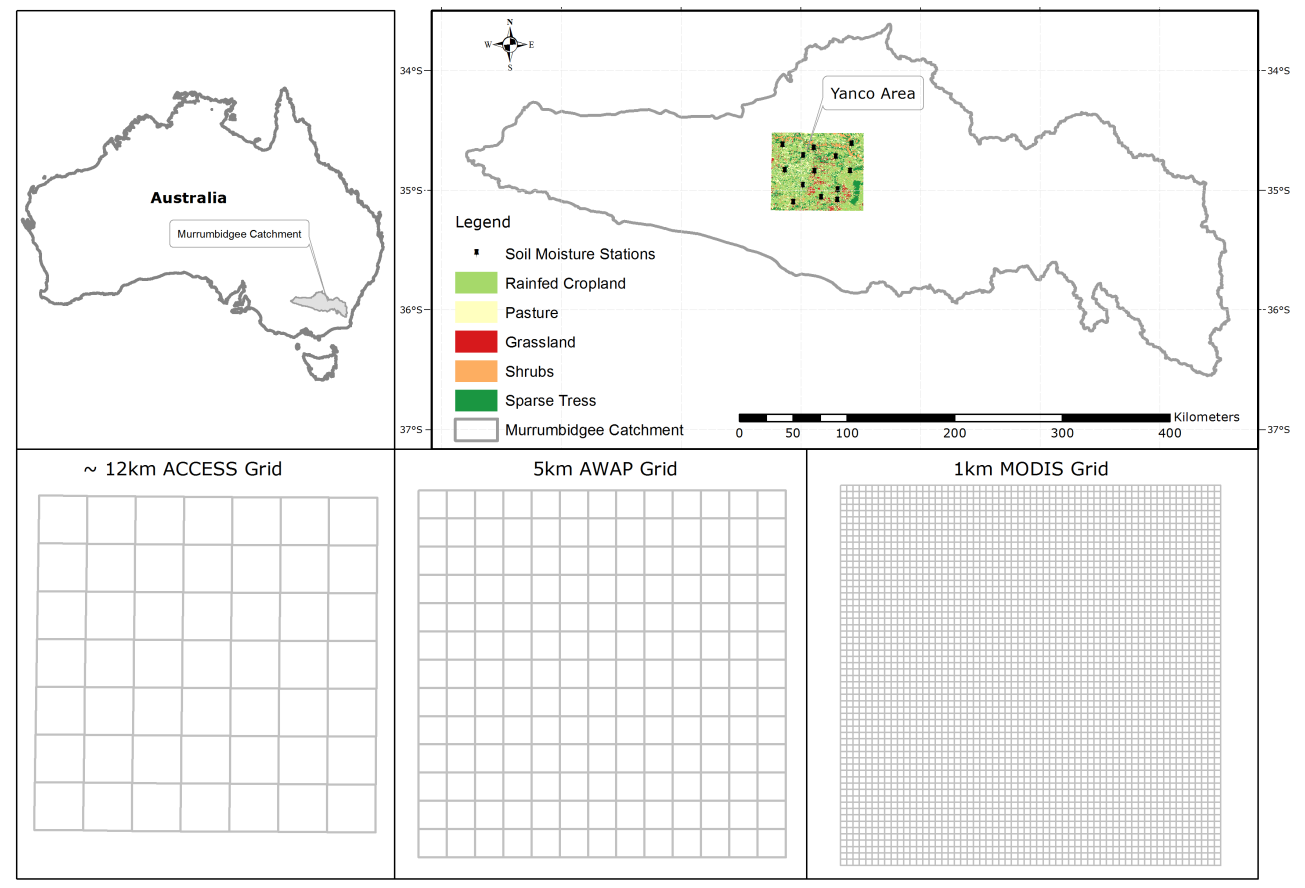

Figure 1: Location of the Yanco region and vegetation data in the study area are presented at the top panel. The bottom panels shows the spatial configuration of the 12-km ACCESS-A, 5-km AWAP, and 1-km MODIS grids in the Yanco area. 
The soil moisture data for the three spatial resolutions, $12-\mathrm{km}, 5-\mathrm{km}$ and 1- $\mathrm{km}$ are land surface model estimates obtained through the Joint UK Land Environment Simulator (JULES). The JULES model is a widely used tiled model of sub-grid heterogeneity which simulates water and energy fluxes between a vertical profile of soil layers, land surface, vegetation, and the atmosphere [Best et al., 2011]. JULES uses meteorological forcing data, surface land cover data, soil information, and values for prognostic variables. The model initialization is conducted for several variables including the temperatures and the liquid and frozen moisture contents of the soil layers; temperature, density, and albedo of the snowpack if present; the temperature and intercepted rain and snow on the vegetation canopy; the temperature and depth of ponded water on the soil surface; and an empirical vegetation growth index. JULES requires the specification of layer thickness of the soil profile, and the proportions for nine land surface categories including broadleaf, needleleaf, grass (temperate and tropical), shrub, urban, inland water, bare soil, and ice-covered surfaces.

The land cover data set used was the Australian National Dynamic Land Cover Data set (DLCD) [Lymburner et al., 2011]. The DLCD was generated from a 16-day Enhanced Vegetation Index composite collected at 250- $m$ resolution from Moderate Resolution Imaging Spectro-radiometer (MODIS) for the period from 2000 to 2008. The DLCD has land cover features clustered into 34 ISO classes with descriptions for the structural character of vegetation, ranging from cultivated and managed land covers (crops and pastures) to natural land covers such as closed forest, and sparse open grasslands. The DLCD data was used to determine the proportion of the nine land cover types for the JULES model.

The soils information was derived from the Digital Atlas of Australian Soils [McKenzie et al., 2000] which was obtained from the Australian Soil Resource Information System (ASRIS). ASRIS provides a digital map of soil types and their descriptions, typical ranges for soil properties for each soil type, morphology, and physical properties of soil profiles. The soil classification system was based on the widely applied Factual Key of Northcote [1979] and later revised to the Australian Soil Classification [Isbell, 2002; Isbell et al., 1997] into 6 textural groups including sands, sandy loams, loams, clay loams, light clays. Soil properties in the Digital Atlas of Australian Soils (DAAS) include information on texture, clay content, bulk density, saturated hydraulic conductivity, and soil layer thickness for horizons A and B [McKenzie et al., 2000; McKenzie and Hook, 1992]. The horizon A data was used to estimate the surface soil information whereas the horizon B data was used to estimate the soil properties for the root-zone soil moisture. The soil properties information are summarized to a $90 \%$ confidence interval by providing the mean/median value, the dispersion of 5 th, and 95th percentile of the average interpreted value.

The meteorological forcing data including short and long wave incoming radiation, air temperature, precipitation, wind speed, pressure, and specific humidity were obtained from Australian Community Climate EarthSystem Simulator - Australian (ACCESS-A) at hourly time step with approximately $12-\mathrm{km}$ spatial resolution [Bureau of Meteorology, 2010]. The ACCESS-A precipitation data was bias corrected using 5- $\mathrm{km}$ gridded precipitation data from the Australian Water Availability Project (AWAP) obtained through the Bureau of Meteorology [Jones et al., 2009, 2007]. The 12- $\mathrm{km}$ AACESS-A data are summarized to represent constituent 5- $\mathrm{km}$ AWAP and the 1-km MODIS grids, such that the overlapping 12- $\mathrm{km}$ data are assigned to the corresponding 5- $\mathrm{km}$ and $1-\mathrm{km}$ grids. It is noted that the input forcing data for all three spatial resolutions are based on the 12-km AACESS-A data, with the precipitation data set bias corrected as stated above. The land cover and the soils data were accordingly summarized to the $12-\mathrm{km}, 5-\mathrm{km}$, and 1- $\mathrm{km}$ grids through spatial overlap, and subsequent determination of the proportions of constituent land cover and soils classes within each grid. The spatial configuration of the 12- $\mathrm{km}$ ACCESS-A grids, the 5- $\mathrm{km}$ AWAP grids, and the 1- $\mathrm{km}$ MODIS grids in the Yanco area are shown in Figure 1.

Using the 12- $\mathrm{km}$ forcing data together with its land cover and soils information, the JULES model was applied to simulate half-hourly soil moisture from January to December 2010. The JULES soil moisture estimation was repeated for the 5- $\mathrm{km}$ and the $1-\mathrm{km}$ grids using their respective forcing, land cover and soil data. The soil moisture was estimated for a soil layer thickness of $0-\mathrm{cm}$ to $30-\mathrm{cm}$ to correspond to the in-situ OzNet soil moisture.

\subsection{Identification of temporally stable locations}

For a pair of spatial resolutions (e.g. $12-\mathrm{km}$ and 5- $\mathrm{km}$ ), the temporal stability of soil moisture was used to determine locations in the smaller resolution (i.e. $5-\mathrm{km}$ ) which best matches the overall soil moisture in the overlapping larger resolution (i.e. 12- $\mathrm{km}$ ). The rationale for this procedure is to identify temporally stable locations in the smaller resolution which can be used to estimate the soil moisture in the larger resolution at future time periods. The temporal stability of soil moisture is usually determined using relative difference 
[Cosh et al., 2008; Martinez et al., 2008; Vachaud et al., 1985] for spatially different locations. But, in the context of soil moisture downscaling, the relative difference is estimated between multiple locations in a smaller resolution and an overlapping area in a larger resolution. As a result, the relative difference for location (or grid) $i$ in the smaller resolution at time $j, \delta_{i j}$ is estimated according to equation 1 .

$\delta_{i j}=\frac{\theta_{j}^{s, i}-\theta_{j}^{b}}{\theta_{j}^{b}}$

where $\theta_{j}^{s, i}$ is soil moisture content in the smaller (denoted s) resolution at location $i$ for time $j$, and $\theta_{j}^{b}$ is the overall soil moisture content in the larger (denoted $\mathrm{b}$ ) resolution for time $j$. The relative difference is summarized by finding the average and its standard deviation for each $i$ location in the smaller resolution. The location in the smaller resolution with the minimum overall relative difference is chosen as the most temporally stable location which can be used to estimate the soil moisture in the larger resolution at future time periods. Note that multiple locations in the smaller resolution may be identified as the most temporally stable locations when their overall relative differences are the smallest available.

\subsection{Relationships between temporally stable locations and physiographic variables}

The temporally stable locations identify which location(s)/grid(s) in the smaller resolution is most representative of the larger resolution, with its standard deviation being equivalent to the expected level of uncertainty. For the purpose of soil moisture downscaling, it is practical to associate the temporally stable locations to landscape physiographic variables such as soil texture and vegetation land cover types. The rationale is to examine whether there are physiographic explanations to temporal persistence of soil moisture, and therefore to facilitate soil moisture downscaling. Using the ASRIS data, the soil texture classes found in the Yanco area are clay, sand, loam, and sandy loam. The vegetation cover types based on the DLCD for the Yanco area are grouped into: broadleaf, needleleaf, and grassland.

To investigate the relationships between temporally stable location and physiographic variables, a similarity indicator was used to determine the level of similarity in physiographic structure between two spatial resolutions (e.g. 12- $\mathrm{km}$ and 5-km) at a time. This requires estimation of the proportion of different categories of soil texture and vegetation types for each of the three spatial resolutions. Note that the proportion for a soil texture category (e.g. sand) or a vegetation type (e.g. broadleaf) for a grid was estimated as the ratio of the area covered by the soil texture group or the vegetation type to the total area of the grid. The similarity indicator $\delta_{p}$ between a grid in the smaller resolution and the overlapping area in the larger resolution was estimated according to equation 2 .

$\delta_{p}=\sum_{m=1}^{n}\left|P_{m}^{s}-P_{m}^{b}\right| / n$

where $P_{m}^{s}$ is the proportion of a physiographic variable of type $m$ in the smaller resolution $s, P_{m}^{b}$ is the proportion of a physiographic variable of type $m$ in the larger resolution $b$, and $n$ is the number of categories for the physiographic variable. A value of $\delta_{p}$ closer to zero indicates a high degree of similarity in physiographic structure whereas a high value of $\delta_{p}$ indicates a high level of dissimilarity.

\section{APPLication RESUlts AND Discussion}

\subsection{Level of temporal stability of soil moisture across spatial scales}

Using the minimum relative difference (according to equation 1) as a basis for selecting temporally stable grids, the soil moisture values for the chosen grids in the smaller resolution were compared to the overlapping grids from the larger resolution in Figure 2. The results show that the soil moisture from the smaller resolution is generally lower than those from the overlapping grid in the larger resolution for all three resolution pairs, as indicated by the negative values of relative difference. The overall root mean square error (RMSE) is very low, about $0.02-\mathrm{m}^{3} / \mathrm{m}^{3}$, along with a very high $R^{2}$ of about 0.8 across the three resolution pairs. The high degree of agreement in soil moisture for the $5-\mathrm{km}$ and $1-\mathrm{km}$ pairing, shows that the level of soil moisture estimation may be related to the small size of their resolutions. The relative extent of the resolution is also a factor for the $12-\mathrm{km}$ estimations where there is a higher degree of agreement with the 5- $\mathrm{km}$ data in comparison to the 1-km data. 


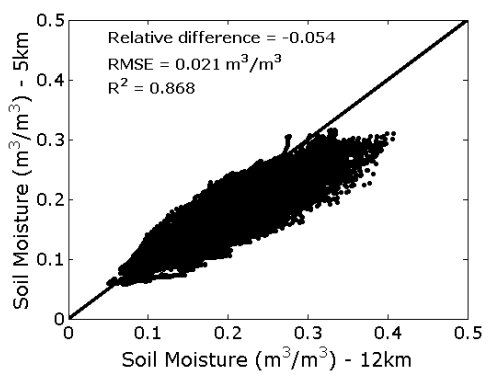

(a) $12-k m$ and $5-k m$ pairing

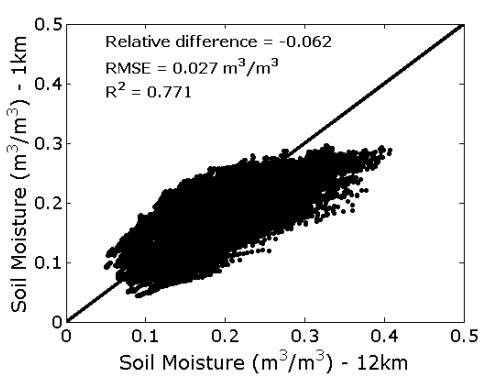

(b) $12-k m$ and $1-k m$ pairing

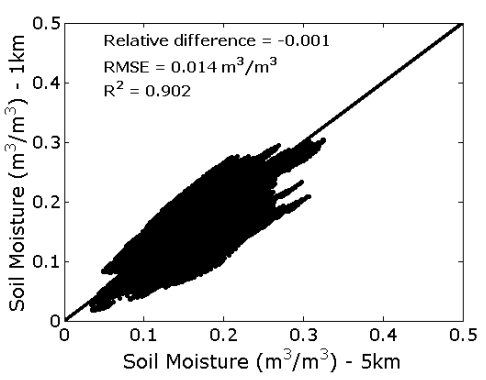

(c) 5-km and $1-k m$ pairing

Figure 2: Overall comparison of soil moisture between temporally stable grids at the smaller resolution with the overlapping grids at the larger resolution across the three different resolution pairs for the top 8-cm soil layer.

\subsection{Temporal stability of soil moisture and its similarity to soil texture}

The selected temporally stable grids are evaluated on the basis of their relationship to the most similar soil texture grids. To evaluate this relationship, the proportion of temporally stable grids which are also most similar in soil texture was determined, along with the proportion of the most similar soil texture grids which are also members of temporally stable grids. These proportions are shown in Table 1 for the three pairs of resolution. The proportions show that soil texture is not a distinguishing factor to identify temporally stable grids, partly due to the high degree of homogeneity in soil texture between several grids in the Yanco area.

Table 1: Relationship between the temporally stable grids (for the top 8-cm soil layer) and the highest soil texture similarity across spatial scales. The middle column shows the proportion of temporally stable grids (in percentage) which also have the highest similarity in soil texture, while the right-hand column shows the proportion of the most similar soil texture grids (in percentage) which correspond to temporally stable grids.

\begin{tabular}{|ccc|}
\hline Pair of resolutions & Temporally stable grids (\%) & Soil texture similarity (\%) \\
\hline $12-k m$ and $5-k m$ & 86 & 88 \\
$12-k m$ and $1-k m$ & 97 & 96 \\
$5-k m$ and $1-k m$ & 98 & 98 \\
\hline
\end{tabular}

\subsection{Temporal stability of soil moisture and its similarity to vegetation cover}

The relationship between the temporally stable grids and similar vegetation cover types was examined by ranking the selected temporally stable grids using the vegetation similarity score. The basis of this procedure is the high degree of heterogeneity in vegetation cover types in the Yanco area compared to soil texture classes. The rank of temporally stable grids based on their vegetation similarity score is presented in Table 2. The ranking values show that similarity in vegetation between resolution has an impact on the soil moisture but is not the only controlling factor.

Table 2: Overall rank of temporally stable grids (for the top 8-cm soil layer) based on similarity of vegetation cover across spatial scales.

\begin{tabular}{|cccc|}
\hline Pair of resolutions & Rank of temporally stable grids & Number of grids & Overall proportion \\
\hline $12-k m$ and $5-k m$ & 5 & 9 & 0.556 \\
$12-k m$ and $1-k m$ & 64 & 130 & 0.492 \\
$5-k m$ and $1-k m$ & 19 & 34 & 0.559 \\
\hline
\end{tabular}

\subsection{Temporal stability of soil moisture and its similarity to soil texture and vegetation cover}

Both soil texture and vegetation were combined to determine their relationship to selecting temporally stable grids. The relative difference values were compared for the temporally stable grids to separate values from the most similar soil texture and vegetation cover in Table 3 . Using the temporally stable grids as the reference with the minimum relative difference values, the similarity in vegetation cover was found to provide a better estimate of relative difference than soil texture for the top $8-\mathrm{cm}$ soil layer across all three resolution pairs.

Table 3: Comparison of the relative difference values for temporally stable grids (for the top 8-cm soil layer), similar soil texture grids, and similar vegetation cover grids. The percentage increase in relative difference values with those from temporally stable grids as reference are presented in parenthesis.

\begin{tabular}{|cccc|}
\hline Pair of resolutions & Temporally stable grids & Similar soil texture & Similar vegetation cover \\
\hline $12-k m$ and $5-k m$ & $-0.054[0 \%]$ & $0.095[75 \%]$ & $0.079[46 \%]$ \\
$12-k m$ and $1-k m$ & $-0.062[0 \%]$ & $0.092[48 \%]$ & $0.090[45 \%]$ \\
$5-k m$ and $1-k m$ & $-0.001[0 \%]$ & $0.040[3900 \%]$ & $0.038[3700 \%]$ \\
\hline
\end{tabular}


Additionally, the selection of grids based on the combined similarity in soil texture and vegetation cover is presented in Table 4. Generally, there is a high correlation, about $90 \%$ between similarities in soil texture and vegetation cover for grids across all three resolution pairs. The chosen grids based on the combined soil texture and vegetation cover show a significant increase in relative difference with temporally stable grids as the reference.

Table 4: Relative difference comparison showing values for temporally stable grids (for the top 8-cm soil layer), the proportion of grids having both the highest similarity in soil texture and vegetation cover, and the overall relative difference for similar soil texture and vegetation cover and its percentage increase shown in parenthesis.

\begin{tabular}{|cccc|}
\hline Pair of resolutions & Temporally stable & \% Soil texture \& vegetation & Soil texture \& vegetation \\
\hline $12-k m$ and $5-k m$ & -0.054 & $90 \%$ & $0.096[77 \%]$ \\
$12-k m$ and $1-k m$ & -0.062 & $98 \%$ & $0.095[53 \%]$ \\
$5-k m$ and $1-k m$ & -0.001 & $86 \%$ & $0.041[4000 \%]$ \\
\hline
\end{tabular}

The three selection methods based on soil texture and vegetation cover is presented in Figure 3 for all three pairs of resolution. Among the three selection methods, similarity in vegetation cover provides the best criterion for selecting grids with minimum relative difference.

\section{Conclusions}

This study has employed the concept of temporal persistence of soil moisture to identify temporally stable locations at a smaller resolution to match an overlapping area at a larger resolution. Temporally stable grids were identified for three pairings of $12-\mathrm{km}, 5-\mathrm{km}$, and $1-\mathrm{km}$ resolutions by determining the minimum rel-

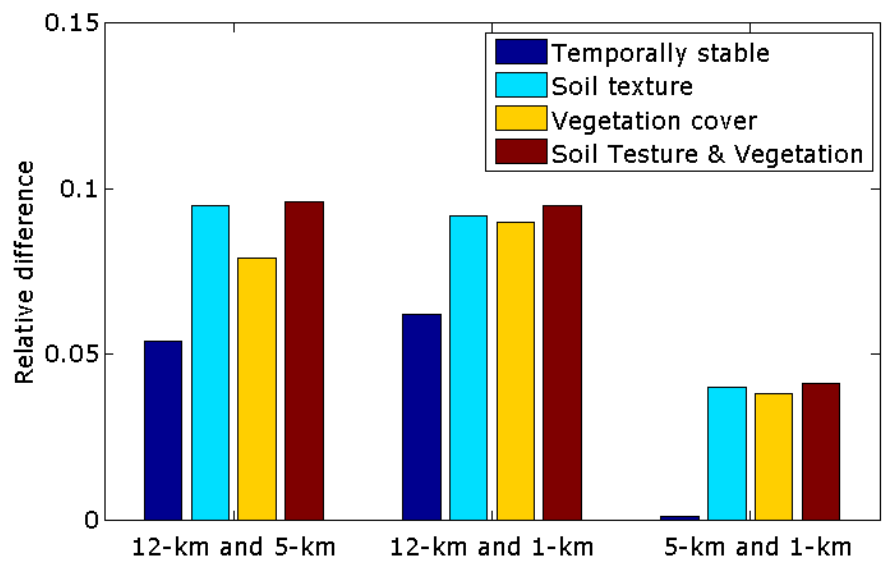

Figure 3: Comparison of the overall relative difference for temporally stable grids, similar soil texture, similar vegetation cover, and the combined soil texture and vegetation cover across the three resolution pairs. ative difference between several locations in a smaller resolution and an overlapping area in a larger resolution. The temporally stable grids were compared to two physiographic indicators based on similarities in soil texture and vegetation cover between locations in the smaller resolution and the overlapping area in the larger resolution.

The temporally stable locations found for the three resolution pairs showed that the percent relative difference range between $6 \%$ and $0.1 \%$; indicating an overall difference of about $3 \%$ of the original value for downscaling soil moisture across the three resolution pairs. The findings showed that rank stability of soil moisture can facilitate soil moisture downscaling, with the capability to improve the accuracy of temporally stable grids for decreasing size of spatial resolution. Soil texture and vegetation cover are not strong indicators of temporally stable locations, with vegetation providing a higher correlation than soil texture due to high degree of homogeneity for soil texture in the Yanco area. Future studies should examine the validation of the chosen temporally stable locations against an independent or out-of-sample period of soil moisture, and in-situ soil moisture. Additional work should investigate the relationships between the temporally stable locations and variable vegetation cover data to account for seasonality of vegetation cover types.

\section{ACKNOWLEDGMENTS}

This work was supported by the Monash University summer research program.

\section{REFERENCES}

Best, M. J., M. Pryor, D. B. Clark, G. G. Rooney, R. L. H. Essery, C. B. Mnard, J. M. Edwards, M. A. Hendry, A. Porson, N. Gedney, L. M. Mercado, S. Sitch, E. Blyth, O. Boucher, P. M. Cox, C. S. B. Grimmond, and R. J. Harding (2011). The Joint UK Land Environment Simulator (JULES), Model description - Part 1: Energy and water fluxes. Geosci. Model Dev. Discuss. 4, 595-640, doi:10.5194/gmdd-4-595-2011. 
G. Dumedah et al., Scale variations associated with downscaling soil moisture

Bureau of Meteorology (2010). Operational implementation of the access numerical weather prediction systems. NMOC Operations Bulletin 83, 1-34.

Chen, Y. J. (2006). Letter to the editor on "rank stability or temporal stability". Soil Science Society of America Journal 70, 306.

Cosh, M. H., T. J. Jackson, S. Moran, and R. Bindlish (2008). Temporal persistence and stability of surface soil moisture in a semi - arid watershed. Remote Sensing of Environment 112, 304 - 313.

Dumedah, G. and P. Coulibaly (2011). Evaluation of statistical methods for infilling missing values in highresolution soil moisture data. Journal of Hydrology 400(1-2), 95102, doi:10.1016/j.jhydrol.2011.01.028.

Isbell, R., W. McDonald, and L. Ashton (1997). Concepts and rationale of the australian soil classification. Technical report, CSIRO, ACLEP Land and Water, Canberra.

Isbell, R. F. (2002). The australian soil classification. Technical report, CSIRO Publishing, Melbourne, Revised Edition.

Jones, D., W. Wang, R. Fawcett, and I. Grant (2007). Climate Data for the Australian Water Availability Project. Australian Water Availability Project Milestone Report, Bur. Met., Australia, 37 pp.

Jones, D. A., W. Wang, and R. Fawcett (2009). High-quality spatial climate data-sets for Australia. Australian Meteorological and Oceanographic Journal 58, 233-248.

Lymburner, L., P. Tan, N. Mueller, R. Thackway, A. Lewis, M. Thankappan, L. Randall, A. Islam, and U. Senarath (2011). The national dynamic land cover dataset (DLCD), record 2011/31, www.ga.gov.au/landcover,. Technical report, Geoscience Australia.

Martinez, C., G. R. Hancock, J. D. Kalma, and T. Wells (2008). Spatio - temporal distribution of near - surface and root zone soil moisture at the catchment scale. Hydrological Processes 22, $2699-2714$.

Martinez Fernandez, J. and A. Ceballos (2005). Mean soil moisture estimation using temporal stability analysis. Journal of Hydrology 312, 28 - 38.

McKenzie, N., D. Jacquier, L. Ashton, and H. Cresswell (2000). Estimation of soil properties using the atlas of australian soils. Technical report, CSIRO Land and Water Technical Report 11/00. Available from http://www.clw.csiro.au/publications/technical2000/.

McKenzie, N. J. and J. Hook (1992). Interpretations of the atlas of australian soils. consulting report to the environmental resources information network (erin). Technical report, CSIRO Division of Soils Technical Report 94.

Northcote, K. (1979). A factual key for the recognition of australian soils. Technical report, Glenside, South Australia: Rellim Technical Publications.

Peischl, S., J. P. Walker, C. Rüdiger, N. Ye, Y. H. Kerr, E. Kim, R. Bandara, and M. Allahmoradi (2012). The AACES field experiments: SMOS calibration and validation across the Murrumbidgee River catchment. Hydrology and Earth System Sciences 16, 1697-1708, doi:10.5194/hess-16-1697-2012.

Singh, R., T. Wagener, K. van Werkhoven, M. E. C. Mann, and R. (2011). A trading-space-for-time approach to probabilistic continuous streamflow predictions in a changing climate accounting for changing watershed behavior. Hydrology and Earth System Sciences (HESS) 15, 3591-3603.

Smith, A. B., J. P. Walker, R. I. Western, A. W.and Young, K. M. Ellett, R. B. Pipunic, R. C.and Grayson, F. H. S. Siriwardena, L.and Chiew, and H. Richter (2012). The murrumbidgee soil moisture monitoring network data set. Water Resources Research 48(W07701), doi:10.1029/2012WR011976.

Vachaud, G., A. Passerat De Silans, P. Balabanis, and M. Vauclin (1985). Temporal stability of spatially measured soil water probability density function. Soil Science Society of America Journal 49, $822-828$.

Western, A. W., R. B. Grayson, and G. Bloschl (2002). Scaling of soil moisture: a hydrologic perspective. Annual Review of Earth and Planetary Sciences 30, 149-180.

Western, A. W., S. L. Zhou, R. B. Grayson, T. A. McMahon, G. Bloschl, and D. J. Wilson (2004). Spatial correlation of soil moisture in small catchments and its relationship to dominant spatial hydrological processes. Journal of Hydrology 286(1 - 4), 113 - 134. 